\title{
Reciprocity Theory of Elementary Particles
}

\author{
MAX BORN \\ University of Edinburgh, Edinburgh, Scotland
}

\section{INTRODUCTION}

$T^{1}$ HE theory of elementary particles which I propose in the following pages is based on the current conceptions of quantum mechanics and differs widely from the ideas which Einstein himself has developed in regard to this problem. I hope that it may nevertheless be acceptable as a contribution to this volume in honor of his 70 th birthday, as it is based on his famous relation between energy $E$ and mass $m$ of a physical system, $E=m c^{2}$, and as it can be interpreted as a rational generalization of his ("special") theory of relativity.

Relativity postulates that all laws of nature are invariant with respect to such linear transformations of space time $x^{k}=(\mathbf{x}, t)$ for which the quadratic form $R=x^{k} x_{k}=t^{2}-\mathbf{x}^{2}$ is invariant (the velocity of light is taken to be unity). The underlying physical assumption is that the 4-dimensional distance $r=R^{\frac{1}{2}}$ has an absolute significance and can be measured. This is a natural and plausible assumption as long as one has to do with macroscopic dimensions where measuring rods and clocks can be applied. But is it still plausible in the domain of atomic phenomena?

Doubts have been expressed a long time ago, e.g., by Lindemann (Lord Cherwell) (14) in his instructive little book. I think that the assumption of the observability of the 4-dimensional distance of two events inside atomic dimensions is an extrapolation which can only be justified by its consequences; and I am inclined to interpret the difficulties which quantum mechanics encounters in describing elementary particles and their interactions as indicating the failure of that assumption.

The well-known limits of observability set by Heisenberg's uncertainty rules have little to do with this question; they refer to the measurements of coordinates and momenta of a particle by an instrument which defines a macroscopic frame of reference, and they can be intuitively understood by taking into account that even macroscopic instruments must react according to quantum laws if they are of any use for measuring atomic phenomena. Bohr has illustrated this by many instructive examples. The determination of the distance $R^{\frac{1}{2}}$ of two events needs two neighboring space-time measurements; how could they be made with macroscopic instruments if the distance is of atomic size?

If one looks at this question from the standpoint of momenta, one encounters another paradoxical situation. There is of course a quantity analogous to $R$, namely $P=p^{2}=p_{k} p^{k}=E-\mathbf{p}^{2}$, where $p_{k}=(\mathbf{p}, E)$ represents momentum and energy. But this is not a continuous variable as it represents the square of the rest mass. A determination of $P$ means therefore not a real measurement but a choice between a number of values corre- sponding to the particles with which one has possibly to do. This is the problem which is now in the center of interest : by estimating $\mathbf{p}$ and $E$ for a particle observed in the Wilson chamber or in a photographic emulsion, one obtains a rough value of the rest mass which may permit one to recognize the kind of particle with which one has to do. If the value of $P$ thus obtained is however incompatible with the known particles a new one is discovered. During the last year this has happened several times, and one gets the impression that there may be no end of it. New types of mesons are found almost every week, and it seems to be not an extravagant extrapolation to suppose that there is an infinite number.

It looks, therefore, as if the distance $P$ in momentum space is capable of an infinite number of discrete values which can be roughly determined while the distance $R$ in coordinate space is not an observable quantity at all.

This lack of symmetry seems to me very strange and rather improbable. There is strong formal evidence for the hypothesis, which I have called the principle of reciprocity, that the laws of nature are symmetrical with regard to space-time and momentum-energy, or more precisely, that they are invariant under the transformation

$$
x_{k} \rightarrow p_{k}, \quad p_{k} \rightarrow-x_{k} .
$$

The most obvious indications are these: The canonical equations of classical mechanics

$$
\dot{x}^{k}=\partial H / \partial p_{k}, \quad \dot{p}_{k}=-\partial H / \partial x^{k}
$$

are indeed invariant under the transformation (1), if only the first 3 components of the 4 -vectors $x^{k}$ and $p_{k}$ are considered. These equations hold also in the matrix or operator form of quantum mechanics. The commutation rules

$$
x^{k} p_{l}-p_{l} x^{k}=i \hbar \delta_{l}^{k},
$$

and the components of the angular momentum,

$$
m_{k l}=x_{k} p_{l}-x_{l} p_{k}
$$

show the same invariance, for all 4 components. These examples are, in my opinion, strongly suggestive, and I have tried for years to reformulate the fundamental laws of physics in such a way that the reciprocity transformation (I,1) is valid (3). I found very little resonance in this endeavor; apart from my collaborators, K. Fuchs and K. Sarginson, the only physicist who took it seriously and tried to help us was A. Landé (13). But our efforts led to no practical results; there is no obvious symmetry between coordinate and momentum space, and one had to wait until new experimental discoveries 
and their theoretical interpretation would provide a clue.

I see now this clue, in the fact mentioned above, that the number of elementary particles is not limited. For this compels us to a change of attitude to the whole problem.

For a long time physics was mainly interested in the electron. Dirac's equation seemed to provide a satisfactory description of its motion with the help of a spinor wave function $\psi$. When nuclear physics became the center of interest, similar wave equations were established for nucleons (neutrons and protons). The electromagnetic phenomena were considered from the same standpoint as a vector wave field describing the motion of photons with the rest mass zero. Then came Yukawa's meson with a similar vector field but a finite rest mass. For each new particle a new field and a new set of field equations were established, containing the rest mass as the only physical parameter. On top of this system of pure fields, one for each kind of particle, one had to construct the mutual interactions of different fields.

This procedure seems now to be wholly inadequate; one cannot continue inventing new field equations for every new type of particle if it is probable that there is an infinite number. There must be a general principle to determine all possible field equations, in particular all possible rest-masses. While in current physics the central problem is to find the wave function $\psi$ from a given set of field equations,

$$
F \psi=0 \text {, }
$$

one has now to assume the attitude that the "field operator" $F$ itself is the unknown, including its characteristic parameter, the rest mass.

I shall show that the principle of reciprocity provides a solution of this new problem-whether it is the correct solution remains to be seen by working out all consequences. But the simple results which we have obtained so far are definitely encouraging (5).

\section{GENERAL WAVE OPERATORS}

The first trivial application of the reciprocity principle consists in the splitting of Planck's constant $\hbar$ into two factors

$$
\hbar=a b,
$$

where $a$ is length, $b$ a momentum. In general considerations the value of $\hbar$ shall be taken to be unity. All coordinates $x^{k}$ will be measured in units $a$, all $p_{k}$ in units $b$, so that from now on $x^{k}$ and $p_{k}$ are dimensionless. The notations of relativity will be used, with the metric tensor

$$
\begin{aligned}
g_{k l}=g^{k l}=0, & (k \neq l) ; \\
g_{11}=g_{22}=g_{33}=-1, & g_{44}=1 ; \\
g^{11}=g^{22}=g^{33}=-1, & g^{44}=1 ; \\
x^{k}=g^{k l} x_{l}, & x_{k}=g_{k l} x^{l} .
\end{aligned}
$$

Particles with integral spin, like photons and certain kinds of mesons, are generally supposed to have a wave function which satisfies a wave equation of the form

$$
P \psi=\kappa^{2} \psi, \quad P=p^{2}=p_{k} p^{k}, \quad p_{k}=i\left(\partial / \partial x^{k}\right),
$$

where the constant $\kappa$ is proportional to the rest mass; going over to customary units one has

$$
\mu=(b / c) \kappa=(\hbar / a c) \kappa .
$$

One can write (II.3) in the form

$$
F(P) \psi(x)=0,
$$

where $F(P)=P-\kappa^{2}$; but one can just as well take for $F$ a function of the form

$$
F(P)=F_{1}(P)\left(P-\kappa^{2}\right),
$$

where $F_{1}(P)=0$ has no roots.

If, on the other hand, $F_{1}(P)$ is itself of the form $F_{2}(P)\left(P-\kappa_{1}^{2}\right)$ then the Eq. (II.5) will have another set of solutions corresponding to particles with the rest mass $\mu_{1}=b \kappa_{1} / c$. Generalizing this one sees that by choosing a proper function $F$ with the roots $\kappa_{1}, \kappa_{2}, \kappa_{3}, \cdots$ one has in (II.5) a wave equation representing simultaneously particles with different rest masses.

This consideration can be easily extended to particles with spin $\frac{1}{2}$, by taking as argument of $F$ instead of $P$ the quantity

$$
\eta=\alpha^{k} p_{k}, \quad \alpha^{k} \alpha^{l}+\alpha^{l} \alpha^{k}=2 g^{k l} .
$$

In this way one obtains a vast generalization of wave mechanics describing any number of particles with different rest masses with the help of one single operator $F$, which is a function of the relativistic invariant $P$ or $\eta$ (where obviously $\eta^{2}=p^{2}=P$ ).

Two problems are now to be solved:

(I) The conservation laws for the field density and for the momentum-energy tensor have to be formulated for this extremely general theory, and the field has to be quantized in such a way, that the particle interpretation is possible.

(II) The much too general formalism has to be reduced and narrowed in such a way that the rest masses are not arbitrary but definite numbers; i.e., one has to find a principle to determine the so far arbitrary function $F$. That is just the problem formulated in the introduction, which I contend to be solved by the principle of reciprocity.

\section{CONSERVATION LAWS AND FIELD QUANTIZATION}

The first problem has been solved by my collaborator, Dr. H. S. Green, who approached it independently from a quite different angle. It had been previously observed by different authors that one can generalize the ordinary wave equation by repeatedly applying the d'Alembertian operator," which is equivalent to the $P$ of the present paper. I have published myself a short note on this question in "Nature" (4), where field equations are derived which are equivalent to the two equations 
$P\left(P-\kappa^{2}\right) \chi=0$ and $P\left(P-\kappa^{2}\right) P \varphi=0$; the point of the paper was that the constant $\kappa$ which appears here as a mass was introduced in the Lagrangian as a coupling constant between the two wave functions $\chi$ and $\varphi$. Podolski (16) has used a similar procedure leading to the first of the above equations and has shown that the elementary solution corresponding to a point charge corresponds to a finite energy. Stueckelberg (18), Bopp (8), Heisenberg (12), Madhava Rao (15), Bhabha (1), and others have attempted similar generalizations of the ordinary wave equation without obtaining more than interesting formal results.

Several authors have attempted to tackle the most general case where the wave equation is of the form (I.5), with an arbitrary $F$, in other words, where the Lagrangian density contains all derivatives with respect to space and time of the wave function. There are papers on this subject by Chang (9) and de Wet (10), but their formalism is very cumbersome. Green (11) attacked the same problem and succeeded in finding a much simpler and very powerful method, which, quite apart from its physical applications, is also of considerable mathematical interest as it represents a new, purely operational approach to the calculus of variations. I was fortunate enough to find this method ready made for my purpose, and to have Green's help in working it out.

I can only sketch Green's method here. Instead of the wave function $\psi(x)$ the statistical operator $\rho$ is used, which for a pure state reduces, in the $x$ representation, to $\psi(x) \psi^{*}\left(x^{\prime}\right)$ but can also be applied to mixture and is then, in this representation a Hermitean matrix $\rho\left(x, x^{\prime}\right)$. A function $F\left(p, p^{\prime}\right)$ of the eight arguments,

$$
p_{k}=i\left(\partial / \partial x^{k}\right), \quad p_{k}{ }^{\prime}=-i\left(\partial / \partial x^{k^{\prime}}\right) ;
$$

is then a differential operator of what is, in general, an infinite order which, when applied to $\rho\left(x, x^{\prime}\right)$, produces an expression linear in $\rho$ and all its partial derivatives. This function

$$
L\left(x, x^{\prime}\right)=F\left(p, p^{\prime}\right) \rho\left(x, x^{\prime}\right)
$$

is called the Lagrangian operator (shortly Lagrangian). It is a relativistic invariant (scalar); but $\rho$ need not be a scalar, but may be a spinor, vector, or tensor of any order. If $\rho$ is e.g., a covariant tensor then $F$ has to be a contravariant tensor of the same order.

The Lagrangian density of the usual theory is the diagonal element obtained by setting $x=x^{\prime}$. For this operation the symbol \langle\rangle shall be used; then the Hamiltonian principle is

$$
\delta \iint\langle L\rangle d \Omega d t=0
$$

where $\Omega$ is the space considered which is usually a big volume tending to infinity so as to cover the whole space. It can easily be seen that the field equations in operational form are simply

$$
F(p) \rho=0, \quad F\left(p^{\prime}\right) \rho=0,
$$

where

$$
F(p)=F(p, p)
$$

the usual form is obtained by taking the diagonal elements,

$$
\langle F(p) \rho\rangle=0 .
$$

If one defines $G^{k}$ and $G^{k^{\prime}}$ by

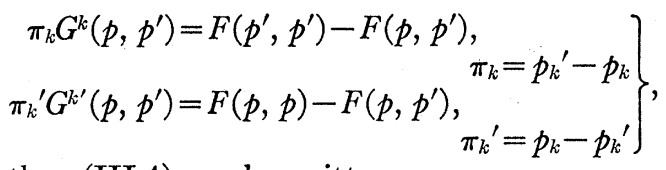

then (III.4) can be written

$$
\left(F+\pi_{k} G^{k}\right) \rho=0, \quad\left(F+\pi_{k}^{\prime} G^{k^{\prime}}\right) \rho=0 .
$$

Subtracting the second from the first, one obtains, since $\pi_{k}+\pi_{k}^{\prime}=0$,

$$
\pi_{k}\left(G^{k}+G^{k^{\prime}}\right) \rho=0,
$$

which is the equation of continuity in operational form, from which one obtains the customary expression by setting $x=x^{\prime}$; for if $A\left(x, x^{\prime}\right)$ is any operator then

$$
\begin{array}{r}
\left\langle\pi_{k} A\right\rangle=-i\left\langle\left(\frac{\partial}{\partial x^{k}}+\frac{\partial}{\partial x^{k^{\prime}}}\right) A\left(x, x^{\prime}\right)\right\rangle \\
=-i\left(\partial / \partial x^{k}\right)\langle A\rangle,
\end{array}
$$

hence from (III.8)

$$
\frac{\partial}{\partial x^{k}}\left\langle\left(G^{k}+G^{k^{\prime}}\right) \rho\right\rangle=0 .
$$

This shows that the 4 vector

$$
R^{k}=\frac{1}{2}\left(G^{k}+G^{k^{\prime}}\right) \rho
$$

represents by its first 3 components the density flux, by its last $R^{4}$ the scalar density. From (III.10) it follows that

$$
d N / d t=0, \quad N=\int\left\langle R^{4}\right\rangle d \Omega
$$

where the integration extends over all space. Hence the total amount of matter is conserved.

Other conservation laws are as easily derived by multiplying the field Eqs. (III.4) first by powers of $p_{k}$ and $p_{k}{ }^{\prime}$ and then proceeding as above. In this way it can be shown that the 4 -vector representing the total momentum and energy

$$
P_{k}=\int\left\langle\frac{1}{2}\left(p_{k}+p_{k}{ }^{\prime}\right) R^{4}\right\rangle d \Omega
$$

is constant in time.

As the field equations are linear, they can be solved by the expression

$$
\begin{aligned}
\rho=\Omega^{-1} \rho_{l j}\left(\mathbf{p}, \mathbf{p}^{\prime}\right) \exp \left\{i\left(\mathbf{p} \cdot \mathbf{x}-E_{l} t\right)\right. & \\
& \left.-i\left(\mathbf{p}^{\prime} \cdot \mathbf{x}^{\prime}-E_{j}{ }^{\prime} t^{\prime}\right)\right\},
\end{aligned}
$$


where $\mathbf{p}, \mathbf{p}^{\prime}, E_{l}, E_{j}{ }^{\prime}, \rho_{l j}$ are constants, provided that $E_{l}$ and $E_{j}^{\prime}$ are solutions of the equations

$$
F(p) \equiv F(\mathbf{p}, E)=0, \quad F\left(p^{\prime}\right) \equiv F\left(\mathbf{p}^{\prime}, E^{\prime}\right)=0 .
$$

There will be in general several roots $E_{l}$ for a given $\mathbf{p}$ and $E_{j}{ }^{\prime}$ for a given $\mathbf{p}^{\prime}$. This becomes obvious by taking into account the fact of relativistic invariance of the Lagrangian which, as said above, implies that $F$ represents a set of spinor or tensor components. Each component must have the form $F=F_{0} g$, where $F_{0}$ is a scalar and $g$ a spinor or tensor of unit modulus which depends only on the direction of the vector $p_{k}$. The scalar factor $F_{0}$ will depend, in the case of a tensor, on

$$
P=p_{k} p^{k}=E^{2}-\mathbf{p}^{2},
$$

and in the case of a spinor on

$$
\eta=\alpha^{k} p_{k}=i \alpha_{4} E-\boldsymbol{\alpha} \cdot \mathbf{p},
$$

so that (III.15) reduced to one of the equations

$$
F(P)=0, \quad F(\eta)=0 .
$$

The roots of the first (algebraic or transcendental) equation $\kappa_{j}^{2}$ present, according to (III.16), (III.17), the squares of the possible rest masses; the same holds for the squares of the characteristic roots of the second (spinor) equation. In the dimensionless units used here the energy belonging to a root is $E_{j}=\left(\mathbf{p}^{2}+\kappa_{j}^{2}\right)^{\frac{1}{2}}$.

The general solution of the field equations is obtained by summing over all possible values of $l, j, \mathbf{p}, \mathbf{p}^{\prime}$.

By substituting (III.14) in (III.12), (III.13) one finds

where

$$
N=\sum_{j} N^{(j)}, \quad P_{k}=\sum_{j} P_{k}^{(j)}
$$

$$
N^{(j)}=\sum \mathbf{p} n_{j}(\mathbf{p}), \quad P_{k}^{(j)}=\sum \mathbf{p} n_{j}(\mathbf{p}) p_{k},
$$

with

and

$$
n_{j}(\mathbf{p})=\Gamma_{j}(\mathbf{p}) \rho_{j j}(\mathbf{p}, \mathbf{p}),
$$

$$
\Gamma_{j}(\mathbf{p})=\frac{1}{2}\left\{G^{4}(\mathbf{p}, \mathbf{p})+G^{4^{\prime}}(\mathbf{p}, \mathbf{p})\right\} .
$$

The Eqs. (III.19) and (III.20) show that the quantities $n_{j}(\mathbf{p})$ must be interpreted as the amount of matter in the field.

However, the atomistic division of matter, the presence of particles, i.e., the fact that the $n_{j}(\mathbf{p})$ are integers, cannot be inferred until second quantization is effected.

Green has reformulated the mathematical procedure for second quantization in strictly operational terms; but as this method leads to the same results as the usual procedure it will not be reproduced here and the reader is referred to the original paper (11). The results are these: One has to split the amplitude $\rho_{l j}\left(\mathbf{p}, \mathbf{p}^{\prime}\right)$ of an harmonic term (III.14) of $\rho$ in a product

$$
\rho_{l j}\left(\mathbf{p}, \mathbf{p}^{\prime}\right)=\sigma_{l}(\mathbf{p}) \sigma_{j}{ }^{+}\left(\mathbf{p}^{\prime}\right),
$$

and to write $\rho$ itself correspondingly in the form

$$
\rho\left(x, x^{\prime}\right)=\sum_{l j} \sigma_{l}(x) \sigma_{j}^{+}\left(x^{\prime}\right),
$$

where

$$
\begin{aligned}
\sigma_{l}(x) & =\Omega^{-\frac{1}{2}} \sum_{\mathbf{p}} \sigma_{l}(\mathbf{p}) \exp \left\{i\left(\mathbf{p} \cdot \mathbf{x}-E_{l} t\right)\right\} \\
\sigma_{j}\left(x^{\prime}\right) & =\Omega^{-\frac{1}{2}} \sum_{\mathbf{p}^{\prime}} \sigma_{j}\left(\mathbf{p}^{\prime}\right) \exp \left\{-i\left(\mathbf{p}^{\prime} \cdot \mathbf{x}^{\prime}-E_{j}^{\prime} t^{\prime}\right)\right\} .
\end{aligned}
$$

Then the commutation laws for the amplitudes $\sigma$ are

$$
\Gamma_{l}(\mathbf{p})\left\{\sigma_{l}{ }^{+}(\mathbf{p}) \sigma_{j}\left(\mathbf{p}^{\prime}\right) \pm \sigma_{j}\left(\mathbf{p}^{\prime}\right) \sigma_{l}{ }^{+}(\mathbf{p})\right\}=\delta_{l j} \delta_{\mathbf{p} \mathbf{p}^{\prime}},
$$

where the + sign holds for Bose-Einstein statistics, the - sign for Fermi-Dirac statistics and $\Gamma_{l}$ is defined by (III.22).

Then it follows by well-known methods that the quantities $n_{j}$ (p) defined by (III.21) are integers. Further it can be shown that $N$ and the different components of $P_{k}$ are all commuting with one another.

These considerations show that for the most general Lagrangian, which is linear in $\rho$, the field can be quantized and therefore regarded as representing a system of particles of different kinds, distinguished by their rest masses and not interacting with one another.

This is a vast extension of the ordinary theory and therefore rather vague in regard to concrete physical results. The next step will consist in contracting it again by a restrictive condition which leads to definite predictions about the possible particles and their rest masses.

\section{THE PRINCIPLE OF RECIPROCITY}

The restricting principle is the postulate of reciprocity. We consider first the case of integral spin. A function $S(x, p)$ of coordinates and momenta which satisfies the symmetry relation,

$$
S(x, p)=S(p,-x)
$$

is a reciprocal invariant. If the $p_{k}$ are here regarded as the operators $i \partial / \partial x^{k \prime}, S$ becomes an operator. A selfreciprocal function $\mathfrak{F}(x)$ is defined as a solution of the eigenvalue equation

$$
S(x, p) \mathfrak{F}(x)=s \mathfrak{F}(x) .
$$

One can just as well regard the $p_{k}$ as numbers and the $x^{k}$ as the operators $-i \partial / \partial p_{k}$. Then it follows from (IV.1) that (IV.2) is still satisfied when $\mathscr{F}(x)$ is replaced by $\mathfrak{F}(p)$.

The property of self-reciprocity can also be expressed by saying that $\mathfrak{F}(x)$ is its own Fourier transform; i.e., for one pair of variables

$$
\mathfrak{F}(x)=(2 \pi)^{-\frac{1}{2}} \int \mathfrak{F}(p) e^{-i p x} d p .
$$

This was indeed the definition of self-reciprocity suggested by Landé and myself (3). It can be easily shown that any solution of (IV.3) satisfies also (IV.2) for an arbitrary invariant $S(x, p)$ and vice versa. The same holds for several pairs of variables $x^{k}, p_{k}$ as long as the geometry is euclidean, i.e., has a positive definite metric 
tensor $g_{k l}$. For the case of quasi-euclidean, non-definite metric of the relativistic space-time one has to replace the Fourier transform of the time variable by a Laplace transform. Therefore the formulation (IV.2) is more convenient for many purposes.

Yet the formulation (IV.3) is important as it shows that the function $S$ is arbitrary; hence one can choose the simplest relativistically and reciprocally invariant function, namely the "metric-operator"

$$
S=x_{k} x^{k}+p_{k} p^{k}
$$

Any eigenfunction of this $S$ satisfies (IV.3), and all solutions of (IV.3) can be obtained by solving the Eq. (IV.2) with this $S$. These eigenfunctions represent all possible self-reciprocal scalars, vectors, and tensors.

Using the notation introduced in the introduction, one finds (IV.4) can be written

$$
S=R+P \text {. }
$$

This is the generalization of relativity required by the inconsistencies of the current theory. The metric invariant $R$, which is a number scalar in $x$ space, is replaced by the sum (IV.5) symmetric in $x$ and $p$ space, the metric operator. For all situations where $P$ is small, i.e., for macroscopic bodies, ordinary relativity holds. But in atomic processes this is not true; the spacemetric is not independent of the momentum metric, both together determine $S$. That the eigenfunctions of $S$ are the field operators $\mathcal{F}$ is a new feature for which no analogy exists in ordinary relativity. This contention can only be justified by its success: one has to determine the solutions $\mathfrak{F}$ and to see whether they provide an explanation of the observable facts.

Before doing so the case of half-integral spin must be considered. Here the symmetry condition (IV.1) must be replaced by

$$
S(x, p)=T^{-1} S(p,-x) T,
$$

where $T$ is a unitary operator not involving $x^{k}$ and $p_{k}$, and the reciprocal of $\mathscr{F}(x)$ is then $T F(p) T^{-1}$. The simplest possible $S$ is

$$
S=\eta+i \gamma \xi, \quad \xi=\alpha_{j} x^{j}, \quad \eta=\alpha^{j} p_{j},
$$

where $\alpha_{j}(j=1,2,3,4)$ are Dirac's spin matrices and

$$
\gamma=\alpha_{1} \alpha_{2} \alpha_{3} \alpha_{4}=\frac{1}{4 !} \epsilon^{j l m n} \alpha_{j} \alpha_{l} \alpha_{m} \alpha_{n},
$$

$\epsilon$ being the totally antisymmetric tensor. For this $S$ the matrix $T$ is given by

$$
T=(1+i \gamma) /(1+i) .
$$

The functions $\mathfrak{F}$ have to satisfy the equation

$$
\mathfrak{F}^{-1} S \mathfrak{F}=s, \quad \text { or } \quad S \mathfrak{F}=\mathfrak{F} s,
$$

where $s$ is an eigensymbol depending only on $\gamma$.

The proofs of these statements can be found in the original paper, where also the solutions of (IV.2) and
(IV.10) are systematically determined. The results will be given in the next section.

These functions $\mathfrak{F}(p)$ can be directly used for writing down the wave equations (III.4). But in order to build up the complete theory one has to know the functions $F\left(p, p^{\prime}\right)$ of two sets of arguments; they are needed to form the Lagrangian and then the energy-momentum tensor. We have first constructed these functions by replacing the arguments $p_{k}$ in $\mathcal{F}(p)$ by the symmetrical combinations $\frac{1}{2}\left(p_{k}+p_{k}{ }^{\prime}\right)$. This leads in fact to a formally coherent theory but has several drawbacks: It leaves the different $F$ functions completely unconnected and furnishes therefore no explanation for the actual occurrence in nature of some of them, while others are not found; and, what is still more decisive, it leads to wrong numerical results.

The interconnection of the different $F$-functions can be obtained by replacing the eigenvalue Eq. (IV.2) by an analogy to the Bloch equation of ordinary quantum mechanics. Let $z, \zeta$ be another pair of conjugate variables satisfying

$$
\zeta z-z \zeta=1
$$

and determine $F(p, z)$ from

$$
S(x, p) F=z \zeta F, \quad \zeta=\partial / \partial z .
$$

Then one has solutions of the form

$$
F=z^{s} \mathfrak{F}(p),
$$

where $\mathcal{F}$ satisfies (IV.2) with the eigenvalue $s$. One can use also the variable $\sigma=-\log z$, then $z \zeta=-\partial / \partial \sigma$ and (IV.12) reads

$$
S(x, p) F=-(\partial / \partial \sigma) F
$$

with solutions of the form

$$
F=e^{-8 \sigma} \mathfrak{F}(p) .
$$

It is natural to assume that $F\left(p, p^{\prime}\right)$ is self-reciprocal with regard to both sets of variables $p_{k}$ and $p_{k}{ }^{\prime}$. My collaborator, Dr. Kai Chia Cheng, has suggested simple products like

$$
F\left(p, p^{\prime}\right)=\mathfrak{F}(p) \mathfrak{F}\left(p^{\prime}\right)
$$

If these are multiplied by the weight factors $e^{-s \sigma}$ with the same $\sigma$, one has a kind of statistical equilibrium corresponding to a "sub-temperature" $\tau=\sigma^{-1}$, where the abundance of a special $\mathfrak{F}$ depends on the numerical value of the corresponding $s$ (to be determined in the next section). In this way the preponderance of the particles with small $s$ values can be understood. Yet it would be premature to enter into this problem in detail.

The equation for the rest masses of quanta is now

$$
F(p, p)=[F(p)]^{2}=0
$$

and has the same roots $\kappa$ as $F(p)=0$, each of the latter appearing twice. In case several $F(p)$ have equal roots, one can take $F\left(p, p^{\prime}\right)$ to be a linear combination of products (IV.16). This happens for instance for photons (zero roots), as will be seen in Section VII. 


\section{DETERMINATION OF THE SELF-RECIPROCAL FUNCTIONS $\mathcal{F}$}

For integral spin one has to solve the Eq. (IV.2) with the simple function $S$ given by (IV.4), or

$$
\left(-\frac{\partial^{2}}{\partial p_{k} \partial p^{k}}+p_{k} p^{k}\right) \mathfrak{F}=s \mathfrak{F} .
$$

This is easily done by using 4-dimensional polar coordinates $P, \theta, \varphi, \omega$ and factorizing $\mathfrak{F}$ into

$$
\mathfrak{F}=F_{k}(P) Y_{k}(\theta, \varphi, \omega),
$$

where $Y_{k}$ is a 4-dimensional harmonic with the eigenvalues $k(k+2),(k=0,1,2, \cdots)$. Then $\mathfrak{F}_{k}$ satisfies

$$
\frac{d^{2} \mathscr{F}_{k}}{d P^{2}}+\frac{2}{P} \frac{d F_{k}}{d P}-\frac{1}{4}\left(1-\frac{s}{P}+\frac{k(k+2)}{P^{2}}\right) \mathscr{F}_{k}=0 .
$$

The solution of this eigenvalue problem (with the boundary conditions that $\mathcal{F}_{k} \rightarrow 0$ for $\left.P \rightarrow \infty\right)$ are

$$
s=2(2 n-k), \quad \mathfrak{F}_{k}=P^{k / 2} e^{-P / 2} L_{n}^{k+1}(P),
$$

where $L_{n}^{k}(P)$ is the $k$ th derivative of the Laguerre polynomial $L_{n}(P)$ of order $n \geqslant k+1$ (associated Laguerre polynomial).

According to (III.18) the rest masses of the possible particles are given by the roots of the equation

$$
\kappa^{k} L_{n}^{k+1}\left(\kappa^{2}\right)=0 .
$$

The theory predicts therefore the existence of photons $\kappa=0$ and of an infinite number of mesons with integral spin given by the roots of the associated Laguerre polynomials.

For half-integral spin the transformation of the Eq. (IV.10) to polar coordinates with the solution of the resulting differential equations is much more involved. It can be effected by a method very similar to Dirac's solution of his wave equation for the hydrogen atom, by using the operator

$$
L=-i \alpha_{j} \alpha_{l} m^{j l}, \quad m_{j l}=x_{j} p_{l}-x_{l} p_{j} .
$$

The result is this. Each $\mathcal{F}$ function consists again of two factors, one depending on $\eta=\alpha_{k} p^{k}$, the other on the

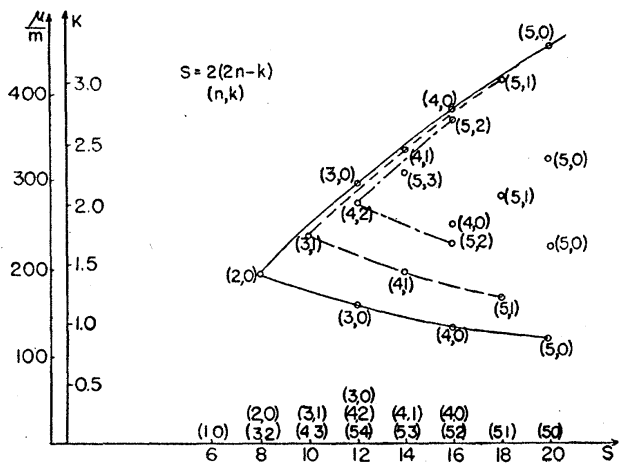

Fig. 1. Meson masses. angles $\theta, \varphi, \omega$; the latter is a generalized spherical harmonic

$$
Z_{k}=(L+4+2 k) Y_{k},
$$

where $Y_{k}$ is the ordinary harmonic.

The factor depending on $\eta$ can best be written separately for the two eigenvalues \pm 1 of $\gamma$. For $k>1$ each of these factors factorizes again into $\eta^{k}$ and a polynomial in $\eta$. The first set of equations for the rest masses is therefore

$$
\eta^{k}=0, \quad k=1,2,3, \cdots,
$$

which represent obviously neutrinos, i.e., particles with rest mass zero and spin $\frac{1}{2}$. The polynomials lead to the equations

$$
\begin{aligned}
\gamma=+1: & \kappa L_{n}^{k+2}\left(\kappa^{2}\right) \pm(n-k-1)^{\frac{1}{2}} L_{n}{ }^{k+1}\left(\kappa^{2}\right)=0, \\
\gamma=-1: & \kappa\left\{L_{n}^{k+1}\left(\kappa^{2}\right)-L_{n}{ }^{k+2}\left(\kappa^{2}\right)\right\} \\
& \pm(n+1)^{\frac{1}{2}} L_{n}{ }^{k+1}\left(\kappa^{2}\right)=0 .
\end{aligned}
$$

The roots of these equations are the masses of an infinite number of mesons with half-integral spin.

All functions $\mathfrak{F}$ with the same $k$ form a tensor of rank $k$ which operates on a tensorial density function.

Numerical values of the reduced rest masses $\kappa$, for integral and half-integral spin are contained in Tables I and II, respectively, at the end of this paper.

\section{MESON MASSES}

The reduced rest masses calculated above are independent of any special assumptions. In order to reduce them to ordinary masses and to compare them with experimental values, one has to identify one of the theoretical particles with an actually observed one of non-vanishing mass; if the reduced mass of the former is $\kappa_{1}$ and the actual mass of the latter $\mu_{1}$, one obtains $a=\hbar / b=\hbar \kappa_{1} / \mu_{1} c$.

Now it has been seen that the theory accounts directly for photons and neutrinos with vanishing rest mass, which are useless for our purpose, and for a great many other particles which are all of the same type and must be obviously identified with mesons whose masses are not very accurately known. It is true that among them are, for high values of $n$ and $k$, particles with very low and very high masses; but none of these has any distinguishing mark which allows the identification with the electron and the nucleon, respectively, whose masses are well known and could be used to fix the absolute length $a$.

It is clear that these permanent particles which differ from the rest by their stability and abundance must be explained in a different way. These considerations lead most naturally back to the old and well-known assumption that the mass of the electron (and also that of the nucleon) is entirely due to electromagnetic self-energy. While this hypothesis has met with insuperable difficulties when applied to ordinary Maxwellian electrodynamics it gives finite and reasonable results in the new theory which will be sketched in the next sections. Here these results will be anticipated. 
It follows from dimensional reasons that the mass of the electron is given by

$$
m=e^{2} / a c^{2}
$$

apart from a numerical factor of order unity. The exact determination of this factor is of course an essential part of the theory and will be treated later. It will be seen that (VI.1) is almost correct.

If it is introduced into (II.4) one obtains for the actual masses

$$
\mu=\left(\hbar c / e^{2}\right) \kappa m=137 \kappa m .
$$

Figure 1 shows meson masses computed from the $\kappa$ values of Table $\mathrm{I}$.

In comparing these results with the experiments one is confronted with the difficulty, that the experimental results are not very precise. It appears to be settled that the most stable meson, the $\mu$-meson, has a mass very near $200 \mathrm{~m}$. That agrees well with the theoretical value for the first particle $(n, k)=(2,0)$ of the series of integral spin with the mass $\sqrt{2} 137 \mathrm{~m}=194 \mathrm{~m}$, which appears also as the first of the series of half-integral spin for $\gamma=-1,(n, k)=(1,0)$.

The measurements of the masses of the $\pi$-mesons (which decay into $\mu$-mesons) are still disputed; the most probable value $\mu_{\pi} / \mu_{\mu}$ seems at present to be 1.35 . There are several theoretical particles which correspond approximately to this value. It seems to be quite possible that the considerable scattering of the experimental values is not only due to the difficulty of measurement but to actually different particles being observed. I prefer to refrain from a definite attempt of identification until the theory is further developed in collaboration with the experimentalists.

Although considerations about the meson masses have first given us some confidence in the theory, I think now that there is another feature of it which provides much stronger evidence, namely the fact that all difficulties connected with the divergence of the expressions for self-energies and interactions (cross sections) disappear without any artificial trick.

\section{THE ELECTROMAGNETIC FIELD}

The following considerations are mainly due to Green who soon will publish a detailed paper on the subject. I can give here only a short sketch of his theory.

The electromagnetic field is described in the usual way by the 4 vector potential $A_{k}(x)$; hence the statistical operator is a tensor of the 2nd order

$$
\rho_{l}^{k}=\frac{1}{2} A^{k}(x) A_{l}\left(x^{\prime}\right),
$$

and the function $F\left(p, p^{\prime}\right)$ must therefore be also a tensor of the second order. According to Cheng's formula (IV.16) one has therefore to take a vectorial $F(p)$; there are only two of these, $(n, k)=(2,1)$ and $(2,0)$, and only the first of these does not involve mesons coupled to the photons. This set $(2,1)$ is in fact the combination with the lowest $s(n, k)=2(2 n-k)$, namely $s(2,1)=6$, which leads to real (zero) rest masses, and should therefore be the most important one.
The Lagrangian of the Maxwell field can be obtained from $F_{l}{ }^{k}=p^{k} p_{l}-\delta_{l}^{k} p^{m} p_{m}$. Hence the correct combination of the self-reciprocal functions $p_{k} e^{-p^{2} / 2}$ is

$$
F_{l}^{k}\left(p, p^{\prime}\right)=e^{-\frac{1}{2}\left(p^{2}+p^{\prime 2}\right)}\left(p^{k} p_{l}{ }^{\prime}-\delta_{l}^{k} p^{m} p_{m}{ }^{\prime}\right) .
$$

It can be supposed that by a gauge transformation the relation

$$
p^{k} A_{k}=0
$$

is ensured (not as in the usual theory, as an expectation value, but identically). Then the field equations are

$$
e^{-p^{2}} p^{2} A_{k}=0 \text {. }
$$

If one expands the exponential factor and retains only the first terms, one obtains Podolsky's equation mentioned in Section III:

$$
\left(1-p^{2}\right) p^{2} A_{k}=0 \text {. }
$$

This however leads to physically improbable consequences, namely the existence of particles of rest mass $b / c$ (in ordinary units) which occur independently but always associated with the electromagnetic field. This difficulty disappears if the Eq. (VII.4) is accepted, since $\exp \left(-p^{2}\right)=0$ has no roots.

Equation (VII.2) has solutions representing plane waves which do not differ from the classical solutions. But it has also solutions with point singularities which differ from the ordinary Coulombian expressions. If by analogy with classical theory a current density 4-vector $j_{k}$ is introduced one has instead of (VII.4)

$$
e^{-p^{2}} p^{2} A_{k}=4 \pi j_{k}
$$

For a point charge at rest one has

$$
j_{1}=j_{2}=j_{3}=0, \quad j_{4}=e \delta(r),
$$

where $\mathbf{r}=\mathbf{x}-\mathbf{x}_{0}$ and $\delta$ is a Dirac symbolic function.

Using ordinary units the solution of (VII.5) for this case, which can be easily obtained by Fourier transformation, reads

where

$$
A_{1}=A_{2}=A_{3}=0, \quad A_{4}=\stackrel{e}{a}-Y\left(\begin{array}{l}
r \\
-
\end{array}\right)
$$

$$
Y(x)=\frac{1}{x} \frac{1}{(\pi)^{\frac{1}{2}}} \int_{0}^{x} e^{-x^{2} / 4} d x .
$$

This modification of Coulomb's law has already been suggested by myself and published in collaboration with Rumer (7) as long ago as 1931, and it appears later sporadically in the literature, as a more or less arbitrary assumption, while in the reciprocity theory it is a necessity. The main features of this potential are these: for $x \gg a$ it goes over in the Coulomb potential, for $x \rightarrow 0$ it tends to the finite value $A_{4} \rightarrow e / a(\pi)^{\frac{1}{2}}$ and it leads to a finite static energy

$$
\begin{aligned}
& m_{0} c^{2}=\frac{1}{2}\left(e^{2} / a\right) \int Y(r / a) \delta(r) d \mathbf{r} \\
& \quad=\left(e^{2} / 2 a\right) Y(0)=\left(e^{2} / 2(\pi)^{\frac{1}{2}} a\right) .
\end{aligned}
$$


But this is not the whole rest energy associated with a charge $e$, in fact only about a third of the total; other important contributions come from the interaction with the radiation field, as will be seen in the next section.

The solution (VII.7) for a charge at rest can be generalized for a moving charge by a Lorentz transformation; the vector $\mathbf{r}=\mathbf{x}-\mathbf{x}_{0}$ has to be replaced by

$$
\tilde{\mathbf{r}}=\left\{\mathbf{v} \times(\mathbf{r} \times \mathbf{v})+\mathbf{v} \frac{\mathbf{r} \cdot \mathbf{v}-v^{2} t}{\left[1-\left(v^{2} / c^{2}\right)\right]^{\frac{1}{2}}}\right\} v^{-2} .
$$

The scalar invariant $r=|\tilde{\mathbf{r}}|$ can be expressed in terms of the angular momentum of the charge about an origin in space-time. The 6-vector angular momentum is defined by

$$
m_{k l}=x_{k} p_{l}^{0}-x_{l} p_{k}{ }^{0}, \quad p_{k}=m v_{k} /\left[1-\left(v^{2} / c^{2}\right)\right]^{\frac{1}{2}} ;
$$

it consists of the two space vectors

$$
\begin{gathered}
\mathbf{m}=m /\left[1-\left(v^{2} / c^{2}\right)\right]^{\frac{1}{2}}(\mathbf{x} \times \mathbf{v}) \\
\mathbf{n}=m c /\left[1-\left(v^{2} / c^{2}\right)\right]^{\frac{1}{2}}(\mathbf{x}-\mathbf{v} t)
\end{gathered}
$$

and the square of its scalar is found to be

$$
\mu^{2}=\frac{1}{2} m_{k} \iota m^{k l}=\mathbf{n}^{2}-\mathbf{m}^{2}=m^{2} c^{2} \tilde{r}^{2} .
$$

The argument of the potentials for a moving charge is therefore

$$
\tilde{r}=\mu / m c^{2} .
$$

The solution of (VII.5) for a moving point charge is then

$$
A_{k}=\left(e_{0} / a c\right) v_{k} /\left[1-\left(v^{2} / c^{2}\right)\right]^{\frac{1}{2}} Y(\tilde{r}) .
$$

A first step in the direction of quantum theory can be made at once. It is well known that in Dirac's theory of the electron the classical velocity vector has to be replaced by the anticommuting Dirac matrices $\alpha^{k}$. If the notation (Eq. (IV.7))

$$
\eta=\alpha^{k} p_{k}, \quad j=\alpha^{k} j_{k}, \quad A=\alpha^{k} A_{k}
$$

is used, the 4 equations (VII.5) can be condensed into one

$$
e^{-\eta^{2}} \eta A=4 \pi j
$$

(because $\eta^{2}=p^{k} p_{k}=p^{2}$ ). This quantity $A$ will be used in the following, as an abbreviation for the $A_{k}$, even if the treatment is classical and no spin matrices are used.

\section{THE ELECTRON}

The theory of the electron developed by Green is a revival of old ideas in modern form; namely, that the electron is a singularity in the electromagnetic field and that its mass is purely electromagnetic. The early attempts to achieve this end failed because of the infinite self-energy of a point charge in a Maxwellian field. Abraham's suggestion of a rigid electron was in contradiction to relativity. Other models of extended electrons needed cohesive forces of non-electromagnetic origin. Therefore modifications of Maxwell's equations were suggested, first by Mie. A non-linear electrodynamics proposed by myself and worked out in collaboration with Infeld, was successful in the frame of classical theory, but resisted all attempts to quantize it, just because of its non-linearity. The new theory based on the principle of reciprocity is linear but preserves nevertheless the advantages of the non-linear theory, namely its unitary character and the finite self-energy of a point electron. I can give here only a brief outline.

Just as in the Born-Infeld theory one has two kinds of field, one directly derived from the potentials

$$
\begin{aligned}
& E_{k l}=(\mathbf{E}, \mathbf{B})=\partial A_{l} / \partial x^{k}-\partial A_{k} / \partial x^{l} \\
&=-\dot{i}\left(p_{k} A_{l}-p_{l} A_{k}\right),
\end{aligned}
$$

the other $D_{k l}=(\mathbf{D}, \mathbf{H})$ obtained by multiplying this (from the left) with $\exp \left(-p^{2}\right)$. Then Maxwell's equation holds in the usual form, and also the energy-momentum tensor can be expressed in the ordinary way.

More important than this formal analogy is the fact that the new theory is unitary. The charges are singularities of the field and inseparable from the field. The usual fiction that the electrons and the photons have an independent existence must be abandoned. In fact neither a set of photons nor a set of electrons is an observable object by itself; the two only become observable by their interaction, and together form a complex system whose internal processes are the objects of experience. There is no essential difficulty in developing the program in the frame of classical theory; but in applying quantum theory some new features appear which Green has worked out. The classical treatment follows the lines of the ordinary Maxwellian theory as closely as possible, the only difference being the exponential factor in $F$.

The proper field of the electron $A^{e}$ is defined by (VII.15) and (VII.16); it is a special solution of (VII.17), or of

$$
F A^{e}=4 \pi j,
$$

where $j$ represents a moving charge. If one calculates

$$
L^{e}=\frac{1}{2} \int\left\langle F A^{e} A^{e^{\prime}}\right\rangle d \Omega
$$

one finds $m_{0} c^{2}\left[1-\left(v^{2} / c^{2}\right)\right]^{\frac{1}{2}}$; this is not the Lagrangian of a moving mass $m_{0}$ but its negative. Hence, if one regards not the $A^{e}$ but the position $\mathbf{x}_{0}$ and velocity $\mathbf{v}$ of the singularity as the unknown function, one has to take $-L^{e}$ as the corresponding part of the Lagrangian. ${ }^{*}$

If $A^{f}$ is the total field its Lagrangian, as functional of $A^{f}$, is

$$
L^{f}=\frac{1}{2} \int\left\langle F A^{f} A^{f^{\prime}}\right\rangle d \Omega
$$

* The negative sign can be understood by remarking that, for a given $j$ the complete Lagrangian which leads to (VIII.2) is

$$
\int\left\{\frac{1}{2}\left\langle F A^{e} A^{e^{\prime}}\right\rangle-4 \pi j A^{e}\right\} d \Omega ;
$$

if $j$ is eliminated with the help of (VIII.2) one obtains $-L^{e}$ where now again $j$ can be regarded as the unknown. 
and the Lagrangian of the interaction is

$$
L^{e f}=-\frac{1}{2} \int\left\langle F\left(A^{e} A^{f^{\prime}}+A^{f} A^{e^{\prime}}\right)\right\rangle d \Omega .
$$

The total field $A^{f}$ also satisfies (VIII.2), but is, of course, a much more general solution; the difference

$$
A^{f}-A^{e}=A^{r}
$$

is the pure radiation field. The total Lagrangian is

$$
L=-L^{e}+L^{f}+L^{e f}
$$

by simultaneous variation of $A^{f}$ and of $\mathbf{x}_{0}, \mathbf{v}$ one obtains the correct field equations and equations of motion. The calculation gives for the latter

$$
\begin{aligned}
\frac{d}{d t}\left(\frac{m_{0} \mathbf{v}}{\left[1-\left(v^{2} / c^{2}\right)\right]^{\frac{1}{2}}}\right. & \left.+e \mathbf{A}_{0}^{f}\right) \\
& =-e\left[\frac{\partial}{\partial \mathbf{x}} \cdot\left(A_{4}^{f}-\frac{1}{c} \cdot \mathbf{v} \cdot \mathbf{A}^{f}\right)\right]_{0}
\end{aligned}
$$

where the index 0 indicates that the function is to be taken at the singularity $\mathbf{x}_{0}$. If one now replaces $A^{f}$ by $A^{e}+A^{r}$ the contributions of the part $A^{e}$ can be worked out explicitly. From (VII.7), (VII.8), and (VII.10), it is seen that $A^{e}$ is an even function of $\tilde{\mathbf{r}}$; hence its derivatives on the right-hand side vanish for $\mathbf{x}=\mathbf{x}_{0}$. On the left-hand side one finds $e A_{0} f=2 m_{0} v / \sqrt{ }\left(1-v^{2} / c^{2}\right)$. The result is

$$
\begin{aligned}
\frac{d}{d t}\left(\frac{m \mathbf{v}}{\left[1-\left(v^{2} / c^{2}\right)\right]^{\frac{1}{2}}}\right. & \left.+e A_{0}{ }^{r}\right) \\
& =-e\left[\frac{\partial}{\partial \mathbf{x}} \cdot\left(A_{4}^{r}-\frac{1}{c} \mathbf{v} \cdot \mathbf{A}^{r}\right)\right]_{0},
\end{aligned}
$$

where

$$
m=3 m_{0}=\lambda\left(e^{2} / a c\right), \quad \lambda=\left[3 / 2(\pi)^{\frac{1}{2}}\right]=0.846 .
$$

Equation (VIII.9) is equivalent to Lorentz' equation of motion.

$$
\frac{d}{d t} \frac{m \mathbf{v}}{\left[1-\left(v^{2} / c^{2}\right)\right]^{\frac{1}{2}}}=e\left(\mathbf{E}^{r}+\frac{1}{c}\left(\mathbf{v} \wedge \mathbf{B}^{r}\right)\right),
$$

where $\mathbf{E}^{r}, \mathbf{B}^{r}$ are the field vectors belonging to the radiation potential $A^{r}$.

Equation (VIII.10) shows that indeed two thirds of the mass are due to the interaction with the field. The numerical factor $\lambda$ is rather near to unity. The quantum treatment will add another small term (transversal selfenergy); there may be further corrections due to the interaction of the electron with mesons.

This quasi-stationary treatment neglects the reaction of the radiation produced by the acceleration of the electron. Green has worked it out in detail and found in first approximation the well-known Lorentz reaction force, proportional to $\partial^{2} v / \partial t^{2}$, but also higher terms. This is in contradiction to Dirac's classical theory of the electron where he maintains that the Lorentz reaction force is exact. Dirac's theory is an example of the socalled "subtraction physics" which evades the difficulties of self-energy and similar problems by declaring them as spurious, produced by unphysical terms in the fundamental equations. I have always rejected this attitude and regarded these difficulties as clues for a deeper understanding of the ultimate laws. I hope that the present theory will justify this attitude.

The quantum treatment of the electron leads to an essential difficulty, namely an apparent contradiction with Heisenberg's principle of uncertainty, as in the expression of $L$ both coordinate $\mathbf{x}_{0}$ and velocity $\mathbf{v}$ of the singularity appear. Green has shown that this objection can be met by a critical inspection of the meaning of the formalism.

The practice of current quantum mechanics, which is to replace the point singularity by a continuous charge distribution $\psi^{+} \alpha_{4} \psi$, where $\psi$ is a solution of Dirac's equation, could not possibly be right in the frame of the unitary theory; for it would lead to the absurd conclusion that the rest energy of the electron depended upon the certainty with which its position is known. This antimony is removed by the remark that one has to distinguish between the intrinsic charge distribution of the electron which is compact, and the probability of finding its center $\mathbf{x}_{0}$ in a given position: the latter is small and uniformly distributed. The only reason that this has not been emphasized before is that the divergence of the self-energy of the electron made the distinction between the relative distribution of charge around the center and the position of the center apparently superfluous.

The formal procedure consists in forming the operator $\Phi=A(r) \psi\left(x_{0}\right)$ and using this in the same way as $A$ before. The current has to be defined by the relativistic generalization of

$$
j=e \delta\left(\mathbf{x}-\mathbf{x}_{0}\right) \psi\left(\mathbf{x}_{0}\right) .
$$

It can then be shown that current quantum electrodynamics remains almost unchanged, with only one important alteration: A factor

$$
\exp \left\{\left(\mathbf{k} \cdot \mathbf{p}_{0}\right)^{2} / E_{0}{ }^{2}-k^{2}\right\} / 2 b^{2}, \quad E_{0}{ }^{2}=m^{2} c^{2}+p_{0}{ }^{2},
$$

has to be added to the matrix elements of the energy of interaction between electrons and photons, namely those matrix elements which represent the emission of a photon with momentum $\mathbf{k}$ by an electron with momentum $\mathbf{p}_{0}$, or the absorption of a photon with momentum $\mathbf{k}$ by an electron with momentum $\mathbf{p}_{0}+\mathbf{k}$.

This "cutting off" factor (VIII.13) is relativistically invariant and removes the infinities appearing in perturbation terms, e.g., the transverse self-energy, but does not affect the high energy tail of actual scattering processes. For instance, the perturbation term for the 
transverse self-energy is the product of two matrix elements, one corresponding to the emission $\mathbf{p} \stackrel{\mathbf{k}}{\rightarrow} \mathbf{p}+\mathbf{k}$, the other to the absorption $\mathbf{p}+\mathbf{k} \stackrel{\mathbf{k}}{\rightarrow} \mathbf{p}$; each contribute the factor $\exp \left[-k^{2} / 2 b^{2}\right]$. Instead of the well-known (divergent) expression $e^{2}(\pi \hbar c m)^{-1} \int k d k$ one has therefore the convergent integral

$$
\begin{aligned}
& \frac{e^{2}}{\pi \hbar c m} \int_{0}^{\infty} \exp \left[-k^{2} / b^{2}\right] k d k \\
&=\frac{e^{2} b^{2}}{2 \pi \hbar c m} \simeq \frac{137}{2 \pi} m c^{2} .
\end{aligned}
$$

This is a rather large value; if one takes into account the theory of holes, one obtains, following Weisskopf, the small value

$$
\frac{e^{2} m c}{2 \hbar} \int_{m c}^{\infty} \exp \left[-k^{2} / b^{2}\right] \frac{d k}{k} \simeq \frac{2 m c^{2}}{137}
$$

In a similar way all perturbation terms corresponding to "roundabout" processes in Heitler's theory become finite and must not be discarded, as Heitler proposes.

On the other hand, one can see that the Klein-Nishina formula is hardly affected by the cutting-off factor, as for the processes corresponding to the perturbation matrix elements the exponent in (VIII.13) becomes zero or at least extremely small $\sim(137)^{-2}$.

For instance, one of the intermediate processes in the scattering mechanism is the absorption of the incident photon with momentum $\mathbf{k}_{0}$,

$$
\mathbf{p} \stackrel{\mathbf{k}}{\longrightarrow} \mathbf{p}+\mathbf{k}_{\mathbf{0}}
$$

\begin{tabular}{|c|c|c|c|}
\hline$n$ & $k$ & $s$ & $\kappa$ \\
\hline 1 & 0 & 4 & \\
\hline 2 & $\begin{array}{l}1 \\
0\end{array}$ & $\begin{array}{l}6 \\
8\end{array}$ & $\begin{array}{l}0 \\
0,1.41\end{array}$ \\
\hline 3 & $\begin{array}{l}2 \\
1 \\
0\end{array}$ & $\begin{array}{r}8 \\
10 \\
12\end{array}$ & $\begin{array}{l}0 \\
0,1.73 \\
0,2.13,2.17\end{array}$ \\
\hline 4 & $\begin{array}{l}3 \\
2 \\
1 \\
0\end{array}$ & $\begin{array}{l}10 \\
12 \\
14 \\
16\end{array}$ & $\begin{array}{l}0 \\
0,2.0 \\
0,1.41,2.45 \\
0,0.97,1.82,2.78 .\end{array}$ \\
\hline 5 & $\begin{array}{l}4 \\
3 \\
2 \\
1 \\
0\end{array}$ & $\begin{array}{l}12 \\
14 \\
16 \\
18 \\
20\end{array}$ & $\begin{array}{l}0 \\
0,2.24 \\
0,1.66,2.70 \\
0,1.20,2.07,3.06 \\
0,0.86,1.60,2.39 .3 .31 .\end{array}$ \\
\hline
\end{tabular}

If the initial momentum of the electron $\mathbf{p}$ is small, its final momentum is $\mathbf{k}_{0}$; when this is introduced for $\mathbf{p}_{0}$ in (VIII.13), together with $\mathbf{k}_{0}$ for $\mathbf{k}$, the exponent becomes, for large $\mathbf{k}_{0}$,

$$
\begin{aligned}
\left(\frac{k_{0}{ }^{4}}{m^{2} c^{2}+k_{0}^{2}}-k_{0}^{2}\right) / 2 b^{2}=- & \frac{m^{2} c^{2}}{2 b^{2}\left[1+\left(m^{2} c^{2} / k_{0}^{2}\right)\right]} \\
& \rightarrow-\frac{1}{2}\left(\frac{m c}{b}\right)^{2} \simeq-\frac{1}{2(137)^{2}}
\end{aligned}
$$

hence the cut-off factor is practically unity.

It seems to me strange that this relativistic cutting-off factor has not been guessed before by one of the many physicists who have followed the subtractive procedures. I think it satisfactory that it comes out of the new theory without any arbitrary assumption, and I regard this as a strong argument in favor of the principle of reciprocity.

\section{THE NUCLEON}

The question now arises how the other type of permanent particles, the nucleons (protons, neutrons), are to be fitted into the theory. Years ago, when working on the non-linear electrodynamics, I published a note in Nature (2) suggesting that the nucleon may be explained as a singularity of the field with angular momentum; the actual rotation with spin 1 combined with the intrinsic spin $\frac{1}{2}$ in the opposite direction give the resultant spin $\frac{1}{2}$, while the magnetic moments (almost) compensate another, and the correct order of the mass is obtained by assuming the self-energy to be due to a magnetic moment just equal one Bohr magneton

$$
\mu_{B}=(e \hbar / 2 m c)=\frac{1}{2}\left(\hbar c / e^{2}\right) e a=\frac{1}{2} 137 e a .
$$

However, it turned out to be impossible to find a rigorous solution of the non-linear field equations corresponding to this state.

In the frame of the present theory this explanation of the nucleon can be obtained by a slight modification of the equations for the electron. The wave function $\psi\left(\mathbf{x}_{0}\right)$ for the center of the electron, introduced in (VIII.12), satisfies, of course, Dirac's equation $\alpha^{k} p_{k} \psi=m c \psi$ which can be written $\left(\alpha^{k} p_{k}+m c\right) \psi=2 m c \psi$. Hence the expression (VIII.12) for the current can also be written in the form

$$
j=(e / 2 m c) \delta(\tilde{r})\left(\alpha^{k} p_{k}+m c\right) \psi .
$$

Here the factors $\delta(\tilde{r})$ and $\left(\alpha^{k} p_{k}+m c\right)$ actually do not commute. It is suggestive to examine the consequences of reversing the order of these factors, so that the current becomes

$$
j=e \delta(\tilde{r})+i(e \hbar / 2 m c) \alpha_{k}\left(\partial / \partial x^{k}\right) \delta(\tilde{r}) .
$$

The singularity consists now of two parts, one being the centrally symmetric one of a point charge, the other the axially symmetric one of a dipole with the moment $\mu_{B}$. The solutions of the field equation for the two singularities are additive. That of the dipole

$$
j=i \mu_{B} \alpha_{k}\left(\partial / \partial x^{k}\right) \delta(\tilde{r})
$$


leads to the self-energy

$$
M c^{2}=\left[3 \mu_{B} / 4(\pi)^{\frac{1}{2}} a^{3}\right],
$$

which agrees with that given in my paper quoted above apart from the numerical factor. If one substitutes in (IX.5) the value of $\mu_{B}$ in terms of $m$ and then $m=\lambda e^{2} / a c^{2}$ one finds

$$
\frac{M}{m}=\frac{3 e^{2} \hbar^{2}}{16(\pi)^{\frac{1}{2}} a^{3} m^{3} c^{2} \lambda^{3}}=\frac{3}{16(\pi)^{\frac{1}{2}}}\left(\frac{\hbar c}{e^{2}}\right)^{2} \frac{1}{\lambda^{3}}
$$

$$
=\frac{3(137)^{2}}{16(\pi)^{\frac{1}{2}}} \frac{1}{\lambda^{3}}=\frac{1986}{\lambda^{3}} .
$$

This shows that the result is very sensitive with respect to the numerical factor $\lambda$. The $\lambda$-value necessary to give the correct experimental ratio $M / m=1846$ is 1.025 . This is in good agreement with the calculation of meson masses, but considerably larger than the theoretical factor (VIII.10), namely 0.846. The discrepancy has still to be cleared up.

All these considerations are preliminary; they are intended to show that the simultaneous existence of two elementary particles with so very different masses as the electron and the nucleon can well be understood from the same mechanism. It cannot be expected that a primitive theory like that suggested here could give exact numerical results. In a comprehensive theory of elementary particles like that which we propose, no particle can be separated from the rest. The self-energy of each particle depends on its interaction with all the others, and not, as we have assumed here, on that of one other type. In particular the nucleons are known to act on one another strongly with meson forces; hence one should expect that a considerable part of their selfenergy must be due to this interaction. The same argument shows that the meson masses as given in Section VI can be only approximations as there will be interactions between them amongst another and the photons. Definite numerical results can therefore be expected only from the completed theory. But this is still far from being achieved. The most conspicuous gap is connected with the fine structure constant or the coupling factor between the singularities and the photon field. Similar factors determine the interactions of nucleons and mesons (mesonic charges). It is clear that these coupling constants must all be treated simultaneously, for one can hardly hope for an explanation of their absolute values until one has a theory of their ratios (e.g., the mesonic coupling factor is about 10 times that of the electronic one). The approach to this question seems to us to lie in considerations as indicated at the end of Section IV, where the different $\mathfrak{F}$ functions are related to one another by weight factors $\exp [-s(n, k) \sigma],(s(n, k)$ $=2(2 n-k)$ for integral spin). It seems to be not impossible that $e^{-\sigma}=z$ may be the fundamental coupling constant, all others being powers of it. My collaborator, Dr. Cheng, is studying this problem in detail.

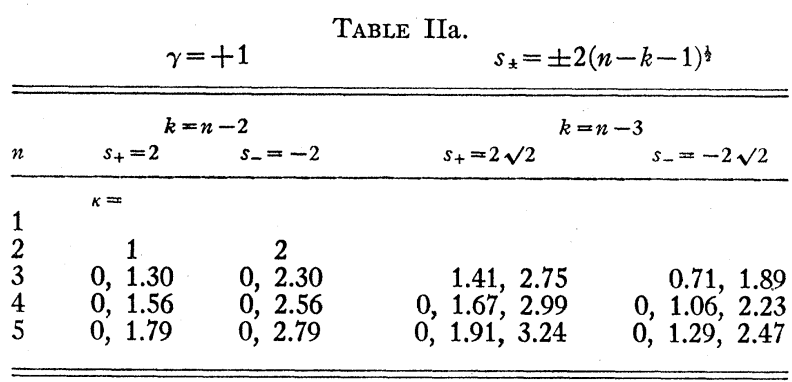

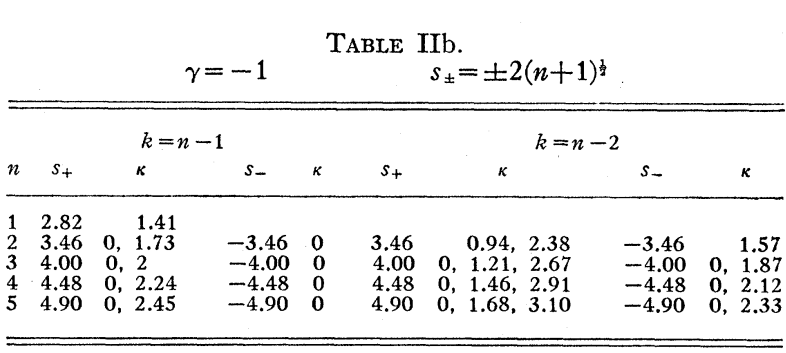

\section{CONCLUSION}

Whether the theory proposed here is the solution of the present difficulties in physics can only be seen by developing all its consequences. One result however can be claimed already:

It is possible to modify the accepted laws in such a way that they form a logically coherent system without infinities.

\section{BIBLIOGRAPHY}

1. H. J. Bhabha, Proc. Ind. Acad. Sci. 21, 241 (1945).

2. Max Born, Nature 136, 952 (1935).

3. Max Born, Proc. Roy. Soc. London A165, 291 (1938); A166, 552 (1938); Proc. Ind. Acad. Sci. 8, 309 (1938); Proc. Roy. Soc. Edinburgh 59, 219 (1938).

4. Max Born, Nature 154, 764 (1944).

5. Max Born, Nature 163, 207 (1949); H. S. Green, Nature 163, 208 (1949).

6. M. Born and K. Fuchs, Proc. Roy. Soc. Edinburgh 60, 100, 141 (1940); K. Fuchs, Proc. Roy. Soc. Edinburgh 60, 147 (1940).

7. M. Born and G. Rumer, Zeits. f. Physik. 69, 141 (1931)

8. F. Bopp, Ann. d. Phys. 38, 345 (1940); 42, 573 (1943).

9. T. S. Chang, Proc. Camb. Phil. Soc. 42, 132 (1946); 44, 76 (1948).

10. J. S. de Wet, Proc. Camb. Phil. Soc. 44, 546 (1948); Proc. Roy. Soc. London A195, 365 (1949).

11. H. S. Green, Proc. Roy. Soc. London A197, 73 (1949); M. Born and H. S. Green, Proc. Roy. Soc. Edinburgh 62, 470 (1949)

12. W. Heisenberg, Zeits. f. Physik. 120, 513, 673 (1943); 123, 93 (1944).

13. A. Landé, Phys. Rev. 56, 482, 486 (1939); J. Franklin Inst. 228, 495 (1939); 229, 767 (1940).

14. F. A. Lindemann, The Physical Significance of the Quantum Theory (Oxford University Press, London, 1932).

15. B. S. Madhava Rao, Proc. Ind. Acad. Sci. A15, 139 (1942); A26, 221 (1947).

16. B. Podolsky, Phys. Rev. 62, 68 (1942); 65, 228 (1944); B. Podolsky and P. Schwed, Rev. Mod. Phys. 20, 40 (1948).

17. K. Sarginson, Proc. Roy. Soc. Edinburgh 61, 77 (1941).

18. E. C. G. Stueckelberg, Nature 144, 118 (1939); Helv. Phys. Acta 14, 51 (1941). 